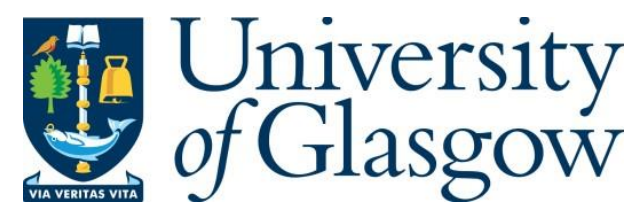

Noble-Jones, R., Thomas, M. J. and Bose, P. (2019) The Lymphoedema Genitourinary Cancer Questionnaire in urology follow-up clinics. International Journal of Urological Nursing, 13(108), pp. 5-12. (doi:10.1111/ijun.12174).

There may be differences between this version and the published version. You are advised to consult the publisher's version if you wish to cite from it.

This is the peer reviewed version of the following article:

Noble-Jones, R., Thomas, M. J. and Bose, P. (2019) The Lymphoedema Genitourinary Cancer Questionnaire in urology follow-up clinics. International Journal of Urological Nursing, 13(108), pp. 5-12, which has been published in final form at 10.1111/ijun.12174. This article may be used for non-commercial purposes in accordance with Wiley Terms and Conditions for Self-Archiving.

\title{
$\underline{\text { http://eprints.gla.ac.uk/168198/ }}$
}

Deposited on: 26 September 2018

Enlighten - Research publications by members of the University of Glasgow http://eprints.gla.ac.uk 


\title{
The Lymphoedema Genitourinary Cancer Questionnaire (LGUCQ) in Urology follow-up
} clinics: original research.

\begin{abstract}
Research Question: Is the Lymphoedema Genitourinary Cancer Questionnaire (LGUCQ) useful to men treated for genitourinary cancer through facilitating symptom disclosure?

Research problem: Lymphoedema can be debilitating and progressive and its association bladder, prostate, testicular and penile cancer, either as a consequence of treatment or progressive disease is well recognised. However, lymphoedema is generally unrecognised during follow-up.
\end{abstract}

Literature review: Research on genitourinary cancer-related lymphoedema is sparse with a lack of reliable prevalence figures. A lack of empirical understanding of the experiences of these men led to the development of the LGUCQ, a simple 2-sided tool to facilitate selfreporting of symptoms and difficulties associated with lymphoedema. Related pilot work suggests that written self-report tools enable men to disclose more sensitive information than they would verbally. However, the LGUCQ had not been formally evaluated in an urooncology department to identify the benefits from the perspective of the patients and health professionals.

Methodology: Thematic analysis of completed LGUCQs and interviews with patients and staff.

Results: Emergent themes included the perceived barriers to symptom disclosure, the LGUCQ as facilitator and pragmatic addition, the support needs of patients and health professionals and refinements required for roll out. Issues limiting identification of 
lymphoedema within uro-oncology services existed. Findings suggest the inclusion of the LGUCQ within uro-oncology clinics could lead to earlier identification of lymphoedema.

Conclusions: Patients could identify genital oedema problems with the LGUCQ increasing prompt and accurate disclosure and normalising the experience.

Funding / Competing interests: Tenovus Cancer Care TIG2017-20; no competing interests.

\section{BACKGROUND}

The management of urological cancers encompasses five different cancer sites - kidney, bladder, prostate, testis and penis. Presentation and management of these cancers are very different. The association of lymphoedema with bladder, prostate, testicular and penile cancer, either as a consequence of treatment or progressive disease is well recognised. It may be that since these cancers are also commonly associated with urinary and sexual dysfunctions the issue of lymphoedema is not considered during follow-up, more research is needed.

The role of the Clinical Nurse Specialist (CNS) in urology is complex and varied. The clinical focus for some is entirely cancer-related, for others it is non-cancer conditions, while others span multiple pathologies (Albaugh 2013; Geldhill et al 2017). Further, whereas some specialist nurses are cancer-site specific e.g. breast cancer, this is not the case for most urooncology CNS roles (Leary et al 2015). The CNS role is essential within the multidisciplinary team (MDT), supporting patient information and enabling shared decision-making at every stage of the cancer journey (Irvine and Chung 2014; Geldhill et al 2017). The informationgiving aspect is significant to the patient experience and understanding of the disease (Leary 
et al 2015). Whilst patient contact with a cancer CNS in England may have increased, studies show worrying regional variances (Leary et al 2015). With increased workload pressures 'meeting information needs' is often reported as being left incomplete (Leary et al 2015). Men with prostate cancer, for example, were found to have continued information needs years after diagnosis (Diver et al 2018). Changing national demographics and increased survival are likely to increase patient numbers and complexity. In this context, priority of attention in daily clinics and in continuous professional development (CPD) is rationally given to conditions of perceived or known risk to mortality or morbidity. However, lower profile conditions like lymphoedema may be overlooked.

Lymphoedema can be congenital or acquired, and may present at any part of the cancer continuum(Bunker 2004; Weinberger et al 2007). The characteristic of swelling (oedema) in the genitals and/or legs and dull ache/discomfort, in absence of other causes (such as thrombosis, pharmaceutical vasodilator or infection), may be the presenting symptom (Turner and Henderson 2011; Hermanns et al 2015). Lymphoedema can be an early postoperative sequela (Villa et al 2017), a longer-term consequence of treatment (Cormier et al 2010; Ragonese et al 2018) or a progressive sign at a palliative care stage (Flynn 2013; Turner and Henderson 2011).

The symptoms of lymphoedema can be debilitating; excessive swelling of the genitals can hinder sexual activity and urination, affect sitting, walking and dressing, and be emotionally disabling (Popovic-Petrovic 2002; Lewis 2004). Ongoing physiological changes cause increased risk of infection, further lymphatic vessel damage and disfigurement (Mortimer 
and Rockson 2014). Identification and treatment by specialist services has been shown to reduce morbidity and complications (Stout Gerich et al 2008; Lacomba et al 2010).

Research on genitourinary cancer-related lymphoedema is sparse compared to breast cancer-related (Cormier et al 2010; Cosgroff and Gordon 2010). A systematic review and meta analysis reported lymphoedema incidence of $21 \%$ in penile cancer, $16 \%$ bladder cancer and $4 \%$ in prostate cancer cases (Cormier 2010), however these figures were based on very small studies. Cancer treatment techniques have continued to improve, but whether this has reduced incidence or, with increased survivorship increased it, remains unknown.

The lack of understanding of the experiences of men with lymphoedema post genitourinary cancer led to the development of the Lymphoedema Genitourinary Cancer Questionnaire (LGUCQ) (Noble-Jones et al 2014). A collaborative development between uro-oncology, lymphoedema specialists and male patients (both with and without lymphoedema) initiated a simple 2-sided tool aimed to facilitate self-reporting of symptoms and difficulties associated with lymphoedema. Related pilot work suggests that written self-report tools enable men to disclose more sensitive information than they would verbally (Sampuro et al 2016). However, the LGUCQ had not been formally evaluated in an uro-oncology department to identify the benefits (or not) from the perspective of the patients and health professionals (urology and lymphoedema). Thus, the following research questions derived from the research literature. 


\section{RESEARCH QUESTIONS}

1. Is the Lymphoedema Genitourinary Cancer Questionnaire (LGUCQ) a useful and pragmatic addition to the patient journey for men treated for genitourinary cancer, from the perspective of both the men and the health care professionals (HCP) involved after cancer treatment?

2. Does the use of the LGUCQ facilitate symptom disclosure for men after treatment for genitourinary cancer in relation to lymphoedema?

3. What support do HCP and patients need in relation to use of the LGUCQ?

\section{SAMPLE}

Male patients of 18 years and over, from 3 Welsh health boards, who had treatment for cancers of the prostate (locally advanced, surgery or radiotherapy to pelvic lymph nodes), bladder (muscle invasive), penis (node invasive treatment or node positive on presentation), or testes (treatment to retroperitoneal lymph nodes or lymph node positive disease) were included; subject to consent.

Ethical approval: NHSREC 17/NW/0492

\section{DATA COLLECTION}

During patient review with a clinician or CNS (late September 2017 - February 2018), men were invited to complete the Lymphoedema Genitourinary Cancer Questionnaire (LGUCQ). Standard information leaflets giving further information regarding lymphoedema were available for patients on completion of the LGUCQ as required. Those men identifying three or more positive responses to the LGUCQ questions were considered for referral by the 
clinician/CNS to the local lymphoedema service. Participating patients, clinicians, CNS and lymphoedema specialists were invited for interview, which could be face-to-face or by telephone, all participants chose to be interviewed by telephone. Interviews took around 20 minutes and with the participant's permission were audio recorded. The lead researcher transcribed the recordings verbatim. Ultimately, interviews took place with eight participants from two health boards: three patients, three lymphoedema practitioners, and only two urology health professionals due to staff availability.

\section{DATA ANALYSIS}

Qualitative interview data was analysed using a thematic framework approach based on the research questions, initially by the primary researcher and then by the second researcher. Any disagreement would be taken to a third member of the team. All research team member were HCPs in urology or lymphoedema. Initial coding of the data was carried out to identify similarities in words and phrasing; subsequent analysis allowed identification of and categorisation of emerging themes within the framework. Further review and discussion of differing interpretation allowed robust conclusions to be drawn. The results of the analysis were then compared against a wider literature on the use of tools for symptom disclosure for a greater conceptual understanding of the issues.

\section{FINDINGS}

Findings are reported in relation to five pragmatic themes:

- perceived barriers to symptom disclosure and information sharing

- the LGUCQ as facilitator of disclosure of lymphoedema symptoms

- the LGUCQ as useful and pragmatic addition to the patient pathway 
- support needed by patients in completing the LGUCQ, or by the health professionals in its administration

- refinements required to the tool, or the process of administration, before wider roll out.

\section{Barriers to Symptom Disclosure and Information Sharing}

At times, the ability to speak openly about symptoms of genital oedema was problematic to both the male patients and the health professionals irrespective if they were male or female. The relational nature of the consultation meant that separating out whether factors were patient or professional may be somewhat unrealistic, but was completed for the purposes of analytical discussion.

Patient quotations are indicated with a $\mathrm{P}$, urology health professionals with $\mathrm{HCP}$, and lymphoedema practitioners with LP.

\section{Approach to the consultation}

Patients described a frank, matter-of fact manner from the professional and themselves as useful, and affect disclosure of symptoms and information sharing.

They were talking about it matter-of-fact to me, and you know, that was fine as far as I was concerned. That brought it out of me... that made me carry on... (P03)

However, there was intimation that this openness was contextual and felt to be a necessity of the situation for patients. 
I can understand why some men wouldn't say about the problem ...the private part area is something that men perhaps generally don't want to talk about... I perhaps wouldn't talk about it normally, but now I've got a problem with it, I have to talk about it. Otherwise people won't understand my problem. (PO2)

There was recognition that symptom specific prompts may be required, but there was an expectation by the HCP that if men had swelling this would be reported.

...[men] perhaps need prompting, so you may have to ask the question, ...but in my experience if men have got swelling, or any issues, they generally tend to be quite open about it. (HCP1)

\section{Lack of risk awareness and surveillance for lymphoedema}

A barrier to early disclosure of lymphoedema symptoms was that the patients were not made aware that they were at risk of lymphoedema and that surveillance for the condition from health professionals was variable. The lack of information on risk led to some men to describe their symptoms of lymphoedema as a shock.

I would have liked to have known more... I wish I'd known beforehand about the lymph system, what the repercussions of the operation could be... I mean the 
swelling came as a shock.... it would have been nice I think if she [the Stoma nurse] had mentioned some of the side effects of the surgery such as lymphoedema... (P01).

I feel that the lymphoedema is out of their [the urologist/oncologist] ball game altogether. They're not entirely aware of what goes on and .... little bit... it's not their problem anymore. ...they've done the prostate bit and then it's on to somebody else. And sometimes I'm not so sure whether they know entirely enough about it all [lymphoedema] you know? (P02)

The variation in levels of surveillance from health professionals was also acknowledged by a HCP participant who recognised that genital oedema was going unrecognised, even within the same hospital or department.

...I know that there is a variation ...that there is a lack of awareness, even in the hospital really. When you see patients from another speciality ...and you think well hang on a minute there is [lymphoedema], ...not all patients are referred...to lymphoedema, they just accept that 'ok your leg is swollen probably because of cancer, but that's it, put up with it'. (HCP4)

\section{Subject avoidance on both sides}

Linked to the enabling effect of specific questions, or prompts, was the timing of enquiry regarding genital oedema, and whether it was the patient or the health professional who delayed broaching the subject. All the lymphoedema practitioners interviewed explained that the subject of genital oedema might not be addressed on the patient's first visit and 
focus would turn to leg oedema if present; the main reason given was a perception of patient shyness. There was some recognition that a more pro-active approach may be required but the delay in addressing the topic of genital oedema was mostly described as a conscious decision on the part of the professional.

...maybe we need to be a bit more pro-active...if I feel the patient doesn't want to talk at that first visit I would leave it until the second visit, and maybe introduce again, being more pro-active in trying to get them to talk about genital or scrotal oedema. (LP2)

A lymphoedema practitioner (LP3) recognised that a reluctance to discuss genital problems may come from either the patient or the professionals. She described how several variables affected the timing and content of the conversation at a first assessment. This included the age of the patient relative to herself, and whether the man was accompanied. Whilst the same lymphoedema practitioner mooted that the gender of the professional in the consultation might also be a factor for men, this was not identified as an issue by the patients interviewed.

One patient (P02) acknowledged a lack of disclosure of genital symptoms but still complained of a delay in his professionals addressing the issue. He suggested that the health professionals were lacking either the experience or the desire to deal with the issue. This may suggest that an additional factor in timely identification of lymphoedema is the training and experience of the health professional to know who is at risk, and that patients may not self-report without direct questioning. 
...seemed to be not really looked at ... And all of that time, things were getting bigger and more difficult in the genital area and... it's almost as if many of them don't normally deal with people like me....And maybe some of them don't want to deal with that problem. (P02)

\section{Summary of findings in relation to first theme:}

The findings support the premise that there are barriers and enablers to disclosure of genital oedema symptoms. Barriers included a lack of risk awareness and surveillance from some health professionals, inappropriate response to patient concern when raised, and subject avoidance by both patient and professional. Enablers included a matter-of-fact, open approach by patients and health care professional and specific prompt questions. The gender of the health care professional was not reported by patients to be a barrier.

The LGUCQ as a tool for facilitating disclosure

\section{A catalyst giving men a voice, symptom validity and control.}

The LGUCQ self-report tool was described as a 'catalyst', which 'triggers off questions' (P01) which enabled and focussed the conversation, validating the relevance of the questions to their condition. The specificity of the questions on the LGUCQ may have helped some patients to earlier identification of symptoms as genital oedema. A lymphoedema practitioner noted that the LGUCQ facilitated the consultation in such a way as to give the patient more control over the discussion. 
I felt it enabled the conversation and made the conversation easier. It allowed the patients to take control and complete the form without feeling judged or feeling pressured to answer any question, [they] were just able to fill it in as they wanted to and then discuss parts of that with me if they needed to ask us. I think it made it a lot easier because ... when they had drawn their diagram themselves, were actually able to talk about specific areas around their genitals with much more ease. (LP1) Similarly, the quality of information in the consultation was reported as being enhanced by the LGUCQ.

He disclosed significantly more to me than... than ever he would have had, ...even if I'd seen him for many more times I don't think he would have disclosed in such detail the psychological impact that his condition has on him. (LP3)

Both patients and professionals valued the production of the tangible self-written record. This was important for some patients, allowing expression of the impact of the condition at that time in their life.

\section{Summary of findings in relation to the second theme:}

The findings support the LGUCQ as a tool, which can act as a catalyst to facilitate disclosure of symptoms of genital oedema through the specificity of the questions. The tool was reported to enable useful conversation and greater disclosure. In addition, both patients 
and health care professional valued the creation of the patient's own written record of the impact of the symptoms, at a given time.

The LGUCQ as a useful addition to the male genitourinary cancer patient pathway All the patients interviewed stated that the questionnaire was acceptable to them. One man (P01) who had already become well informed about lymphoedema reported initially feeling he was completing the LGUCQ for someone else rather than for his own benefit; despite this, he later described it as an 'essential' addition to the care pathway. Patients and health professionals expressed that the LGUCQ self-report tool would add value to the patient care pathway. The reasons given were both practical and psychological.

\section{A normalising effect for men}

The effect of having a questionnaire directed at their particular issues led one patient (P02) to describe it as a 'fantastic thing' and how 'reassuring' it was 'that somebody is actually looking at this and actually asking the questions...'. Another (P01) felt so strongly about the pertinence of the tool that they added further comment to their transcribed interview, suggesting that it might make men feel less alone in their suffering.

I think your questionnaire is essential for male patients following surgery in case they develop lymphoedema; that the format is just right, and all the questions are relevant. I only wish I had been given such a questionnaire at the outset in 2012. I 
would not have felt like an only one and that there was a system to assist in managing the problem. (P01; additional post-interview comment)

Better use of time and resources by generating quality information

A urology-based health care professional (HCP1) pointed out that lymphoedema was not their speciality therefore the specificity of the questions provided a useful aide memoire in the busy outpatient/clinical context. The same HCP pointed out that, in addition to the utility of the LGUCQ for gathering appropriate information, it could save time and resources by doubling as a referral document to specialist lymphoedema services. For the lymphoedema specialists, the LGUCQ was suggested to be a useful supplement to standard lymphoedema assessment documentation. Earlier disclosure of issues related to genital oedema, including psychological problems, was noted to be a product of the use of the LGUCQ.

\section{Facilitating parity with other long-term effects of cancer treatment}

Urology-based health professional (HCP4) identified the LGUCQ as useful in raising the awareness among other health professionals to the risk of lymphoedema for some men and the existence of specialist services for onward referral. In addition, that when patients were aware of their condition, and of the existence of specialist services, they could help drive their own care. This was compared to the commonly acknowledged possibility of impotence and incontinence as consequences of genitourinary cancer and its treatment. 


\section{Summary of findings in relation the third theme:}

the findings support the LGUCQ as a useful and pragmatic addition to the pathway of care of men with genitourinary cancer. The LGUCQ was acceptable to patients, helped normalise their situation and was anticipated to reduce the sense of being feeling alone in the experience. Health care professionals reported the use of LGUCQ led to better use of time and resources through generating timely and better-quality information. Finally, it was recognised as having the potential to raise awareness among other health professionals and patients to bring parity of care with other long-term effects of genitourinary cancer.

Support needed by HCP and patients to use the LGUCQ.

During the study, patients were given time to complete the LGUCQ in a clinic with a health professional nearby. This was the preferred setting for most participants. At interview, the patients reported that they had not needed support to complete the tool but would welcome greater opportunity to discuss any issues raised e.g. P02; this was confirmed by the health professionals. One lymphoedema practitioner (LP1) felt that it would be better to send the LGUCQ out before the appointment, so the patient would have time to think about the questions before attending.

Health care professionals in urology clinics identified support needs for initial adoption of the LGUCQ into regular practice. These included a launch session to describe locally available lymphoedema services, points of contact and how the more urgent referrals might be advanced (HCP1). In addition, that initially there should be wall-posters reminding HCP which patients are at higher risk of lymphoedema and therefore offered the LGUCQ. The 
difficulty of remembering new practice in a busy clinic or of applying unconscious bias in patient selection was identified by one urology HCP (HCP4).

the patients would have been happy enough to fill it in, we just didn't do it, for various reasons, busy clinic, lack of personnel...we didn't give out the questionnaire to all the patients that would have been eligible ...it's just that, sometimes if you think that 'no, this patient is not going to have lymphoedema', you don't give it out..., you start self-selecting patients. (HCP4).

The lymphoedema practitioners interviewed were very experienced and reported needing no support but they anticipated that younger colleagues, or those with less experience, might need further training/support to deal with the questions or emotions raised.

Summary of findings in relation to the fourth theme:

Patients completing the LGUCQ in a clinic environment needed very little support in completing the LGUCQ but wanted time to discuss any issues raised. Health care professionals in urology suggested specific launch information being available when the LGUCQ came into wider practice and visual prompts such as posters on which patients are at risk of lymphoedema. Lymphoedema practitioners felt younger or newer colleagues may need further training or support to deal with the patient issues raised by the LGUCQ. 
Refinements required to the tool, or the process of administration, before wider roll out.

The findings suggested that LGUCQ was well received overall, as was the process of giving it to patients in urology outpatient clinics when they fulfilled the criteria of being at-risk of lymphoedema.

[The LGCQ] was quite well received. The patients were interested. ...It's all very selfexplanatory. I liked the fact that there was also a leaflet to give to the patient as well [standardised national lymphoedema leaflet] ..that's really helpful. So ...they would know and understand a little more about lymphoedema. (HCP1)

Some suggestions for change were made by patient and health professional participants, but none of these were unanimous. These included the location of being given the selfreport tool, having more space to write an explanation of symptoms or impact, and as indicated in the previous section, having more time to discuss the highlighted problems in clinic.

In relation to the location of patients being given the LGUCQ, one patient (P01) suggested that the LGUCQ (and details of their nearest lymphoedema clinic) should be in the discharge pack of the surgical ward for men within the lymphoedema-risk criteria. However, in selfcontradiction, he added that '...some patients will be shy or reluctant to complete the questionnaire, so it could be completed with a professional clinician who will be able to interpret the questions if need be' (P01). Similarly, another patient (P02) and some health professionals thought men should have time to consider the questions for a while before 
attending clinic. However, both the patient and professionals suggested that patients may then not complete it at all or may forget to bring it with them.

One lymphoedema practitioner (LPO3) suggested the LGUCQ could be repeated at review appointments to help map progress or report change of symptoms, so that treatment continues to be patient value-based.

The suggestion of having a digital online completion version of the LGUCQ was acknowledged as being potentially useful but all the patient participants in this study stated a preference for the paper version.

\section{Summary of findings in relation to the fifth theme:}

The LGUCQ was well received by patients and health professionals. It was important to patients and health professionals that patients had time to consider the questions and to discuss any issues raised, but whether the LGUCQ should be completed at home or in a clinic was not clearly established. The potential of the LGUCQ as an ongoing monitoring tool and the relative benefits of paper and digital versions of the LGUCQ were raised and would need further research.

\section{DISCUSSION}

This study had two implicit assumptions, first that there were barriers to the identification of lymphoedema in uro-oncology clinics, second that enabling patient identification of symptoms might overcome such barriers and lead to early, appropriate onward referral. 
The increased recognition of a 'risk of lymphoedema' as a consequence of some cancer treatments has not been seen in relation to genitourinary cancers as it has in breast cancer care. Consequently, the gap widens between these cancer sites in terms of lymphoedema related research and evidence to guide practice. Incidence and prevalence figures for genital oedema remain sparse and identification of those at risk of genitourinary cancerrelated lymphoedema is largely theoretical. Large scale sharing of data has the potential to improve knowledge of genitourinary cancer-related lymphoedema as it has done for penile cancer (Turner and Henderson 2011).

In the absence of specific audited standards or clinical guidelines, and given the complexity and size of urology CNS caseload (Albaugh 2013; Leary et al 2015), it is unlikely that the CNS will initiate a discussion with the patient regarding genital oedema or lymphoedema. It may be that the onus should be on the patients to report troubling symptoms. However, a limiting factor would be that some men, particularly older men, have been found to be reluctant to discuss and disclose problems of a genital nature, affecting their initial healthseeking behaviours (Fish et al 2015; Medina-Perucha et al 2017). Whilst a pilot study by Sampuro et al (2016) found that men with prostate cancer were able to disclose more sensitive information in written than verbal form, this study indicated that using a tool which had patient generated images and vocabulary, the LGUCQ, enabled earlier and more accurate disclosure and helped normalise the experience.

Using objects as tools to enable difficult conversations is a technique common to work with children (NSPCC 2018), adults after trauma (Williamson et al 2018) and with vulnerable adults (Talking Mats 2018). The potential of a co-produced questionnaire to act as a conversation catalyst was the basis for this study and the findings confirmed this premise. 
The study was successful in achieving its objectives in terms of establishing the usefulness of the LGUCQ as a pragmatic addition to the genitourinary cancer care pathway for men at risk of lymphoedema. This was found to be possible without further refinement of the LGUCQ, on the proviso that in terms of support, patients were given the time to discuss any issues disclosed with a health professional who was well-informed in relation to lymphoedema. The findings suggest that some younger or less experienced lymphoedema practitioners might need training, specific to urology and genital oedema problems, in order to feel confident in addressing patient concerns. Given the size and scope of this study, this finding requires further research.

A suggested development of the LGUCQ was a non-cancer version. Lymphoedema practitioners raised that there are men who attend lymphoedema services with non-cancerrelated genital lymphoedema therefore omitting the word 'cancer' would be useful for this wider group of men. The frequency of occurrence is thought to be as many as $10 \%$ of men with bilateral leg oedema (Fischer 2002). There is currently no standard protocol to assess the presence of genital oedema in this patient population, further research is needed. In contrast to evidence reviews in relation to initial health seeking behaviours of men (Fish et al 2015; Medina-Perucha et al 2017) this study did not find gender differences to disclosure; the men reported being just as likely to disclose symptoms of genital oedema to female HCP as to male HCP. This may be because the patient participants were further on in their treatment pathway and had already accommodated to discussion of genital issues with female health professionals. Indeed the men interviewed in this study acknowledged that being diagnosed with a genitourinary cancer had meant that they had adjusted to an increased level of discussion of their genitals because of their specific context. A matter-of- 
fact attitude by health professionals (male and female) was indicated as enabling for patients and, whilst humour with close friends may have been helpful to patients, it was not mentioned as an enabler when used by health professionals. This reflects the earlier work of Chapple and Ziebland (2004) in relation to testicular cancer patients.

The pragmatic design of this study enabled men attending post-cancer treatment urology clinics to complete the LGUCQ. The increased alertness of urology clinic staff, during the period of the study, to men who may be at risk of lymphoedema was acknowledged to be likely a problem to maintain in the long-run. Means of electronically flagging those at risk may be worthy of exploration. The study raised the awareness of lymphoedema practitioners and urology HCP to areas of potential practice improvement. For lymphoedema practitioners the richness of the additional disclosure stimulated by the LGUCQ indicated potential service efficiency by addressing issues earlier in a series of consultations and in recognising when onward referral might be appropriate e.g. to psychological support. For staff in urology clinics, lymphoedema was recognised as only one of a very large number of issues that they may need to address with this group of patients at review, so the simple but specific questions on the 2-sided LGUCQ was appreciated. A limitation of this study was that, without local mandate, such as a clinical standard to identify men at risk of lymphoedema hence prompting the use of the LGUCQ, the busy daily pressures of the urology review clinics often drowned out the study. Setting such a standard and auditing against it with the LGUCQ could further establish its utility to both patients and urology professionals.

Further practical limitations on the study emerged from the current pressures on all UK health services. Over the period of the study several staffing issues occurred which were 
outside the control of the research team. One consultant withdrew due to overwhelming work demands and two CNS changed their posts. These meant that recruitment of patients to the study was frequently broken. Ultimately only two of the three health boards participated.

Despite being a small study, the LGUCQ self-report tool for men was found to be a pragmatic and useful addition to the patient care pathway for a specific and identifiable group of men at risk of lymphoedema. Implementation into regular practice would require very little change to practice, or the tool itself, and the benefits of earlier, appropriate management of symptoms would benefit patients and health service in terms of reduced morbidity. The limited size of the study is acknowledged as a limitation in terms of making wider generalisations but in terms of the research objective to show the pragmatic utility of the LGUCQ self-report tool, the study was successful. Further, that if clinical standards were set this narrow criteria of men after genitourinary cancer i.e. those with lymph node or bladder wall involvement, the next stage could feasibly be a urology-based clinical audit, rather than a scaling up of this specific study.

\section{CONCLUSIONS AND IMPLICATIONS FOR PRACTICE}

The findings of this study suggest that the inclusion of the LGUCQ self-report tool within uro-oncology clinics could improve patient outcomes by the earlier identification of lymphoedema. This albeit small study, found issues which limited the identification of lymphoedema within uro-oncology services from health professionals regardless of gender. The findings also suggested that younger or less experienced lymphoedema practitioners may need additional training in order to feel confident in addressing these issues with 
patients. Thus, placing the onus on patients to identify problems through the LGUCQ may increase a more prompt and accurate disclosure as well as normalising the experience.

\section{WHAT WE KNOW ABOUT THIS TOPIC}

Association of lymphoedema with four of the five urological cancer sites (bladder, prostate, testicular and penile cancer), either as a consequence of treatment or progressive disease is well recognised. However, as these cancers are also commonly associated with urinary and sexual dysfunctions, the issue of lymphoedema is generally unrecognised during follow-up. Little research has been published into the impact of providing a self-report tool for lymphoedema, such as the LGUCQ, to genitourinary cancer patients.

\section{WHAT THIS PAPER ADDS}

This paper adds some understanding to the current gap in the literature surrounding genitourinary lymphoedema. This research that giving patients undergoing genitourinary cancer treatment the opportunity tom complete the LGUCQ may be a prompt and catalyst for enabling difficult conversations. The paper also suggests that health professionals may need additional training in genital oedema to accurately identify and feel more confident in addressing this issue with genitourinary cancer patients. 


\section{REFERENCES}

Albaugh JA, (2013) Urology nursing practice educational preparation, titles, training, and job responsibilities around the globe: Replication. International Journal of Urological Nursing ; 7(2): 85-91

Bunker CB, (2004) Male Genital Skin Disease; London: Saunders.

Chapple A, Ziebland S, (2004) The role of humor for men with testicular cancer. Qualitative Health Research; 14 (8): (1123-1139).

Cormier JN, Askew RL, Mungovan KS, Xing Y, Ross, MI and Armer, JM. (2010) Lymphedema beyond breast cancer. Cancer; 116: 5138-5149.

Cosgriff N, Gordon S, (2010) Cancer-related lymphoedema in males: a literature review. Journal of Lymphoedema 5 (4): 49-61.

Diver S, Avalos G, Rogers ET, Dowling M, (2018) The long-term quality of life and information needs of prostate cancer survivors. International Journal of Urological Nursing; 12 (1): 16-26.

Fischer M, Wohlrab J, Fiedler E, Marsch W. (2002) Genital lymphedema. H\&G Zeitschrift fur Hautkrankheiten; 77: 70-74

Fish JA, Pritchard I, Ettridge K, Grunfeld EA, Wilson C. (2015) Psychosocial factors that influence men's help-seeking for cancer symptoms: a systematic synthesis of mixed methods research. Pchycooncology; 24 (10):1222-1232. 
Flynn K, (2013) Metastatic castrate-resistant prostate cancer: a discussion of the physical and psychological effects. International Journal of Urological Nursing; 7(2): 98-105.

Geldhill R, Allchorne P, Green J, Cornford P, (2017) Knowledge gaps in prostate cancer treatment between physicians and clinical nurse specialists. International Journal of Urological Nursing; 12 (1): 5-8.

Hermanns T, Kuk C, Zlotta A,(2015) Clinical presentation, diagnosis and staging. In: Nargund VH, Raghavan D, Sandler HM, (eds) Urological Oncology. $2^{\text {nd }}$ edition. London: Springer.

Irvine J, Chung SF (2014) Treatment decision-making for early prostate cancer - what can nurses do. Nursing and Health; 2: 23-29

Lacomba MT, Sanchez MJY, Goni AV, Merino DP, del Moral OM, Tellez EC, Mogollon EM. (2010) Effectiveness of early physiotherapy to prevent lymphoedema after surgery for breast cancer: randomised, single blinded, clinical trial. British Medical Journal; $\mathbf{3 4 0}$ (b5396): 1-8

Leary A, Brocksom J, Endacott R, Fleure L, Howdle F, Masterton M, O'Connor A, Swift A, Trevatt P, Aslet P. (2015) The specialist nursing workforce caring for men with prostate cancer in the UK. International Journal of Urological Nursing; 10 (1): 5-13 Lewis M, (2004) Genital lymphedema. A collection of lymphedema papers. Lymphedema People website. http://www.lymphedemapeople.com/thesite/lymphedema_genital.htm (accessed 29/07/2018). 
Medina-Perucha L, Yousaf O, Hunter MS, Grunfeld EA. (2017) Barriers to medical helpseeking among older men with prostate cancer. Journal of Psychosocial Oncology; 35 (5) :531-543.

Mortimer PS, Rockson SG, (2014) New developments in clinical aspects of lymphatic disease, The Journal of Clinical Investigation, 124 (3):915-921.

https://www.jci.org/articles/view/71608 (accessed 29/07/2018).

Noble-Jones R, Fitzpatrick B, Sneddon MC, Hendry DS, Leung HY, (2014) Development of the Lymphoedema Genito-Urinary Cancer Questionnaire. British Journal of Nursing. 23 (18): S14-S19.

NSPCC (2018) Talking about difficult topics.[website]

https://www.nspcc.org.uk/preventing-abuse/keeping-children-safe/talking-aboutdifficult-topics (accessed 11/09/2018)

Popovic-Petrovic S, Nedeljkovic M, Petrovic T, Vasovic M. (2002) Physical treatment of secondary lymphedema of the arm in breast cancer patients. Archive of Oncology; 10: 261-262.

Ragonese M , Salgarello M , Di Gianfrancesco L , Palermo G , Racioppi M , Bassi P, (2018) Incidence and risk factors for lymphedema in Patients underwent radical cystectomy for bladder cancer evaluated with lymphoscintigraphy. European Urology Supplements; 17(4): e2081

Sampuro F, Ruseckaite R, Millar JL,(2016) Comparison of patient-reported quality-of-life and complications in men with prostate cancer between two modes of administration. Clinical Genitourinary Cancer; 14 (4): 284-9. 
Stout Gerich N L, Pfalzer L A, McGarvey C, Springer B, Gerber LH, Soballe P. (2008) Preoperative assessment enables the early diagnosis and successful treatment of lymphedema. Cancer; 112: 2809-2818

Talking Mats 2018 Assisting Vulnerable People to Communicate [website] https://www.talkingmats.com/assisting-vulnerable-people-to-communicate (accessed $11 / 09 / 2018)$

Turner B, Henderson S, (2011) Penile cancer: an update. International Journal of Urological Nursing; 5 (3): 97-106.

Villa G, Bresciani M, Boarin M, Manara D. (2017) The impact of oral nutrition in patients after radical cystectomy: an overview. International Journal of Urological Nursing; 11 (3): 136-143.

Weinberger LN, Zirwas MJ, English JC III. (2007) A diagnostic algorithm for male genital oedema. Journal of the European Academy of Dermatology and Venereology; 21: 15662.

Williamson, V., Hiller,R.M.,. Meiser-Stedman, R., Creswell, C., Dalgleish, T., Fearon, P., Goodall, B., McKinnon, A., Smith, P., Wright, I., Halligan, S.L,(2018) The Parent Trauma Response Questionnaire (PTRQ): development and preliminary validation, European Journal of Psychotraumatology, 9:1. 